\title{
A NOTE ON BRANCH POINTS OF MINIMAL SURFACES
}

\section{ROLF SCHNEIDER}

1. Introduction and formulation of result. One of the classical formulations of the problem of Plateau is as follows:

Given a Jordan curve $\Gamma$ in $n$-dimensional Euclidean space $E^{n}(n \geqq 3)$, determine a real vector function $X(u, v)=\left(x_{1}(u, v), \cdots, x_{n}(u, v)\right)$, defined for $u^{2}+v^{2} \leqq 1$, with the properties:

(a) $X(u, v)$ is continuous for $u^{2}+v^{2} \leqq 1$ and harmonic for $u^{2}+v^{2}<1$;

(b) $E=G, \quad F=0$ in $u^{2}+v^{2}<1$, where $E=X_{u} \cdot X_{u}, \quad F=X_{u} \cdot X_{v}$, $G=X_{v} \cdot X_{v}$ (the subscripts denoting partial differentiation);

(c) by the equation $X=X(u, v)$ ( $X$ position vector with respect to some cartesian coordinate system in $\left.E^{n}\right)$ the circle $u^{2}+v^{2}=1$ is mapped topologically onto $\Gamma$.

If the conditions (a), (b), (c) are satisfied by the function $X(u, v)$, the surface $S$, given by the equation $X=X(u, v), u^{2}+v^{2}<1$, is called a generalized minimal surface of the type of the disc, bounded by $\Gamma$. A singular point of the surface $S$, i.e. a point where $E G-F^{2}=0$, and hence $X_{u}=X_{v}=0$, is called a branch point of order $m$ of the minimal surface, if all the partial derivatives of $X(u, v)$ with respect to $u$ and $v$ vanish at this point up to and including the order $m \geqq 1$, while at least one of the derivatives of order $m+1$ is different from the zero vector. It is easy to see that the branch points must be isolated. The classical solutions of Plateau's problem leave the question open whether, for a given Jordan curve $\Gamma$, there is always a generalized minimal surface of the type of the disc, bounded by $\Gamma$, which is free of branch points. Therefore statements are of interest, which give information about the possibly existing branch points of any generalized minimal surface of the type of the disc, bounded by a Jordan curve $\Gamma$, as soon as certain geometric properties of $\Gamma$ are known. In this sense we have:

ThEorem. Let $\Gamma$ be a Jordan curve with total curvature $\kappa(\Gamma)$ in $E^{n}$. If a generalized minimal surface of the type of the disc, bounded by $\Gamma$, has branch points $\left(u_{i}, v_{i}\right)$ (with $\left.u_{i}^{2}+v_{i}^{2}<1\right), 1 \leqq i \leqq k$, of orders $m_{i}$ respectively, then

$$
1+\sum_{i=1}^{k} m_{i} \leqq \frac{\kappa(\Gamma)}{2 \pi}
$$

Received by the editors March 24, 1966. 
Especially, there cannot be a branch point if $\kappa(\Gamma)<4 \pi$; and there are only finitely many branch points, if $\kappa(\Gamma)$ is finite.

For an analytic Jordan curve this inequality (in a somewhat more general form) is due to Sasaki [6] (compare J. C. C. Nitsche [2], $[3$, p. 236]). Nitsche $[3$, p. 236] has asked for statements about the general case. In the following we shall use an argument of Radó [4] to prove the theorem for arbitrary Jordan curves, thus giving a partial answer to one of Nitsche's problems on minimal surfaces (Problem 27 in [3, p. 258]). The total curvature $\kappa(\Gamma)$ of a closed curve $\Gamma$ is defined in the general case, according to Milnor [1], by $\kappa(\Gamma)$ $=\sup \{k(I I)\}$, where the supremum is taken over all closed polygons inscribed in $\Gamma$, and where $\kappa(\Pi)$ for a closed polygon $\Pi$ is defined as the sum of its exterior angles. (For closed curves of class $C^{\prime \prime}$ this definition leads to the usual total curvature $\int_{\Gamma}\left|X^{\prime \prime}(s)\right| d s$ ( $s$ arc length).)

2. Proof of the theorem. Let $K=\left\{(u, v) ; u^{2}+v^{2}<1\right\}$, let $\partial K$ be the boundary of $K$ and $\bar{K}=K \cup \partial K$. Let the nonconstant real function $h(u, v)$ be continuous in $\bar{K}$ and harmonic in $K$. A point $\left(u_{0}, v_{0}\right) \in K$ is called a critical point (of the function $h(u, v)$ ) of order $\geq m$, if at $\left(u_{0}, v_{0}\right)$ all the partial derivatives with respect to $u$ and $v$ vanish up to and including a certain order $m \geqq 1$. Let $h \mid \partial K$ be the restriction of the function $h(u, v)$ to $\partial K$. We want to show that $h \mid \partial K$ has at least $1+\sum_{i=1}^{k} m_{i}$ different relative maxima on $\partial K$, if there are $k$ different critical points $\left(u_{i}, v_{i}\right) \in K, 1 \leq i \leq k$, of orders $\geqq m_{i}$, respectively.

Lemma 1. If $\left(u_{\alpha}, v_{\alpha}\right) \in K$ is a critical point of order $\geqq m_{\alpha}$, then there are (closed) Jordan arcs $J_{\alpha 0}, J_{\alpha 1}, \cdots, J_{\alpha 2 r-1}$ with $r \geqq 1+m_{\alpha}$ contained in $\bar{K}$ with the properties:

(a) $J_{\alpha i} \cap J_{\alpha j}=\left\{\left(u_{\alpha}, v_{\alpha}\right)\right\}$ for $i \neq j ;\left(u_{\alpha}, v_{\alpha}\right)$ is an endpoint of $J_{\alpha i}$, $0 \leqq i \leqq 2 r-1$;

(b) $J_{\alpha i} \cap \partial K=\left\{\left(u_{\alpha i}, v_{\alpha i}\right)\right\}, 0 \leqq i \leqq 2 r-1$; the points $\left(u_{\alpha 0}, v_{\alpha 0}\right),\left(u_{\alpha 1}\right.$, $\left.v_{\alpha 1}\right), \cdots,\left(u_{\alpha, 2 r-1}, v_{\alpha, 2 r-1}\right)$ are endpoints of the corresponding arcs; they follow each other in this order if $\partial K$ is run through in positive sense;

(c) for $(u, v) \in J_{\alpha i}-\left\{\left(u_{\alpha}, v_{\alpha}\right)\right\}$ inequality $(-1)^{i}\left[h(u, v)-h\left(u_{\alpha}, v_{\alpha}\right)\right]$ $>0$ holds, $0 \leqq i \leqq 2 r-1$.

The proof may easily be seen from an argument of Radó[4, p. 793].

Lемма 2. If the different points $\left(u_{i}, v_{i}\right) \in K, 1 \leqq i \leqq k$, are critical points of orders $\geqq m_{i}$, respectively, then there are $m \geqq 1+\sum_{i=1}^{k} m_{i} m u$ tually disjoint, simply connected open domains $G_{1}, \cdots, G_{m}$ contained in $K$ with the properties: 
(a) For $(u, v) \in K-\bigcup_{j=1}^{m} G_{j}$ and $(u, v) \neq\left(u_{i}, v_{i}\right), 1 \leqq i \leqq k$, the inequality $h(u, v)<\max _{1 \leqq i \S k}\left\{h\left(u_{i}, v_{i}\right)\right\}$ holds;

(b) $\partial K \cap \partial G_{j}$ contains in its (nonempty) relative interior (with respect to $\partial K)$ a point where $h \mid \partial K$ has a relative maximum, $1 \leqq j \leqq m$.

Proof. We proceed by induction. First let $k=1$. Let $J_{10}, \cdots$, $J_{1,2 r-1}$, with $r \geqq 1+m_{1}$, be the Jordan arcs belonging to the critical point $\left(u_{1}, v_{1}\right)$ according to Lemma 1 , and let $\left(u_{10}, v_{10}\right), \cdots,\left(u_{1,2 r-1}\right.$, $\left.v_{1,2 r-1}\right)$ be the corresponding endpoints on $\partial K$. Because of properties (b) and (c) in Lemma 1, the continuous function $h \mid \partial K$ must have a relative maximum between $\left(u_{1 i}, v_{1 i}\right)$ and $\left(u_{1, i+2}, v_{1, i+2}\right)$ for odd $i$, $1 \leqq i \leqq 2 r-1$ (where $\left.\left(u_{1,2 r+1}, v_{1,2 r+1}\right):=\left(u_{11}, v_{11}\right)\right)$. Therefore the connected components $G_{1}, \cdots, G_{r}$ of $K-U_{2\}_{i}} J_{1 i}$ have the properties demanded in Lemma 2, which hence is true for $k=1$.

Suppose now the statement of Lemma 2 is proved for a $k \geqq 1$. Let $K$ contain, then, the $k+1$ critical points $\left(u_{i}, v_{i}\right), 1 \leqq i \leqq k+1$, of orders $\geqq m_{i}$, respectively. After appropriate renumbering, if necessary, we may assume $h\left(u_{k+1}, v_{k+1}\right) \geqq \max _{1 \leqq i \leqq k}\left\{h\left(u_{i}, v_{i}\right)\right\}$. By the inductional hypothesis, to the $k$ points $\left(u_{i}, v_{i}\right), 1 \leqq i \leqq k$, there belong $s$ domains $G_{1}, \cdots, G_{s}$, where $s \geqq 1+\sum_{i=1}^{k} m_{i}$, with the properties of Lemma 2 . Because of property (a), the point $\left(u_{k+1}, v_{k+1}\right)$ is contained in one of these domains, $\left(u_{k+1}, v_{k+1}\right) \in G_{1}$, say. Let $J_{k+1,0}, \cdots, J_{k+1,2 r-1}$, where $r \geqq 1+m_{k+1}$, be the Jordan arcs belonging to $\left(u_{k+1}, v_{k+1}\right)$ according to Lemma 1. Since for $j$ even, $(u, v) \in J_{k+1, j}-\left\{\left(u_{k+1}, v_{k+1}\right)\right\}$ implies the inequality $h(u, v)>h\left(u_{k+1}, v_{k+1}\right) \geqq \max _{1 \leqq i \leqq k}\left\{h\left(u_{i}, v_{i}\right)\right\}$, the arc $J_{k+1}, j$ with $2 \mid j$ must be contained (one endpoint excluded) in $G_{1}$. The components, containing the point $\left(u_{k+1}, v_{k+1}\right)$, of the pointsets $J_{k+1},{ }_{j} \cap G_{1}$, with $2 \nmid j, 1 \leqq j \leqq 2 r-1$, separate the domain $G_{1}$ in to $r$ simply connected domains $G_{1}^{\prime}, \cdots, G_{r}^{\prime}$, each containing (except for its endpoints) one arc $J_{k+1, j}$ with $j$ even. Each endpoint different from $\left(u_{k+1}, v_{k+1}\right)$ of such an arc is contained in a subarc of $\partial K$ each of whose endpoints either is an endpoint of an arc $J_{k+1, j}$, with $2 \nmid j$, or a point of $\partial\left(\partial K \cap \partial G_{1}\right)$. Since $h(u, v)<h\left(u_{k+1}, v_{k+1}\right)$ for $(u, v) \in \partial\left(\partial K \cap \partial G_{1}\right)$ it is clear that the function $h \mid \partial K$ must have a relative maximum in the relative interior (with respect to $\partial K$ ) of $\partial K \cap \partial G_{i}^{\prime}, 1 \leqq i \leqq r$. Hence the domains $G_{1}^{\prime}, \cdots, G_{r}^{\prime}, G_{2}, \cdots, G_{s}$, the total number of which is $r+(s-1)$ $\geqq 1+m_{k+1}+\sum_{i=1}^{k} m_{i}$, have the properties demanded in Lemma 2 .

Thus we have proved Lemma 2 and hence the fact that $h \mid \partial K$ must have at least $1+\sum_{i=1}^{k} m_{i}$ different relative maxima on $\partial K$, if $K$ contains $k$ different critical points $\left(u_{i}, v_{i}\right)$ of orders $\geqq m_{i}$, respectively.

Now let $\Gamma$ be a Jordan curve in $E^{n}$, and let $S$ be a generalized minimal surface of the type of the disc (in the representation given 
initially), bounded by $\Gamma$. Let the different points $\left(u_{i}, v_{i}\right), u_{i}^{2}+v_{i}^{2}<1$, $1 \leqq i \leqq k$, be branch points of $S$ of orders $m_{i}$, respectively. Let $Y$ be a unit vector. The point $\left(u_{i}, v_{i}\right) \in K$ is a critical point of order $\geqq m_{i}$ of the function $h(u, v)=Y \cdot X(u, v)$, which is continuous in $\bar{K}$ and harmonic in $K$. Hence, for the number $\mu(\Gamma, Y$ ) (which may be $\infty$ ) of relative maxima of the function $Y \cdot X \mid \partial K$ we have $\mu(\Gamma, Y)$ $\geqq 1+\sum_{i=1}^{\boldsymbol{k}} \quad m_{i}$. Since $Y$ was arbitrary, the crookedness $\mu(\Gamma)$ $=\min \{\mu(\Gamma, Y)\}$ (where $Y$ ranges over all unit vectors) of $\Gamma$ satisfies the inequality $\mu(\Gamma) \geqq 1+\sum_{i=1}^{k} m_{i}$. But according to Milnor [1, p. 253], the inequality $\kappa(\Gamma) \geqq 2 \pi \mu(\Gamma)$ holds; hence our theorem is proved.

\section{REFERENCES}

1. J. W. Milnor, On the total curvature of knots, Ann. of Math. 52 (1950), 248-257.

2. J. C. C. Nitsche, (Review of [6]), Math. Rev. No. 492, 25 (1963), 104.

3. - On new results in the theory of minimal surfaces, Bull. Amer. Math. Soc. 71 (1965), 195-270.

4. T. Rado, The problem of the least area and the problem of Plateau, Math. Z. 32 (1930), 763-796.

5. - On the problem of Plateau, Springer, Berlin, 1933.

6. S. Sasaki, On the total curvature of a closed curve, Japan J. Math. 29 (1959), $118-125$.

University OF Frankfurt/M, GERMANY 